\title{
Revascularização miocárdica em pacientes com idade igual ou superior a 70 anos
}

Januário M. SOUZA*, Marcos F. BERLINCK*, Myriam G. MOREIRA*, José Renato M. MARTINS*, Maria Cássia S. MOREIRA*. Paulo A. F. OLIVEIRA*, Dante F. SENRA*, Sérgio Almeida de OLIVEIRA*.

SOUZA, J. M.; BERLINCK, M. F.; MOREIRA, M. G.; MARTINS, J. R. M.; MOREIRA, M. C. S.; OLIVEIRA, P. A. F.; SENRA, D. F.; OLIVEIRA, S. A. - Revascularização miocárdica em pacientes com idade igual ou superior a 70 anos. Rev. Bras. Cir. Cardiovasc., 5(3): 141-148, 1990.

RESUMO - A cirurgia de revascularização miocárdica em pacientes acima de 70 anos vem tornandose cada vez mais freqūente. Em 1979 , ela representou $3,3 \%$ dos pacientes operados, alcançando $14,8 \%$ em 1989. Entre janeiro de 1979 e outubro de 1989, dos 7003 pacientes revascularizados, 492 pacientes estavam na oitava ou nona década de vida. A indicação cirúrgica tem sido empregada, preferencialmente, para pacientes com lesōes de alto risco e muito sintomáticos, estando $84 \%$ em grupos III e IV de angina. Quanto ao número de artérias com lesōes críticas, $62,3 \%$ tinham lesōes triarteriais, $31,4 \%$ lesōes biarteriais e $6,2 \%$ lesōes uniarteriais; $15 \%$ dos pacientes tinham lesāo do tronco da coronária esquerda. Operaçōes associadas à revascularização miocárdica foram realizadas em 54 pacientes, sendo 30 aneurismectomias do ventrículo esquerdo, 21 cirurgias valvares e três endarterectomias de carótida. Vinte e oito pacientes estavam na fase aguda do infarto do miocárdio e 27 pacientes estavam sendo operados pela segunda vez. A idade dos pacientes estava compreendida entre 70 e 74 anos em $354(71,9 \%)$ pacientes, entre 75 e 79 em $121(24,5 \%)$ pacientes e entre 80 e 87 anos em $17(3,4 \%)$. A mortalidade imediata (hospitalar ou 30 dias) foi de $8,5 \%$ (42/492) sendo de $2,5 \%$ (162/6511) em pacientes abaixo de 70 anos ( $p<0,0001$ ), operados no mesmo período. De 410 pacientes idosos submetidos apenas à revascularização miocárdica (angina estável e instável), faleceram $21(5,1 \%)$. De 28 pacientes operados na fase aguda do infarto do miocárdio, $13(46,4 \%)$ faleceram, e, de 54 pacientes com operaçōes associadas, oito $(14,8 \%)$ faleceram $(p<0,001)$. Dos 27 pacientes reoperados, houve $5(18,5 \%)$ óbitos. O seguimento tardio pós-operatório variou de dois a 127 meses. Apenas $17(3,9 \%)$ pacientes não puderam ser contactados recentemente. Nesse período, ocorreram 32 óbitos, sendo $15(46,8 \%)$ de causa cardíaca.

DESCRITORES: miocárdio, revascularizaçāo, pacientes idosos.

\section{INTRODUÇĀO}

A população idosa no Brasil vem aumentando consistentemente ${ }^{12}$. As doenças cardiovasculares e, especialmente, a cardiopatia isquêmica são as principais causas de óbito nessa faixa etária. Com - desenvolvimento da cirurgia cardíaca, a revascularização miocárdica foi estendida progressivamente a esse grupo de pacientes. Neste trabalho, analisaremos os resultados obtidos em 492 pacien- tes com idade igual ou superior a 70 anos, operados nos últimos 10 anos.

\section{CASUISTICA E MÉTODOS}

Entre janeiro de 1979 e outubro de 1989 , foram submetidos à revascularizaçăo miocárdica 7003 pacientes, dentre os quais $492(7,02 \%)$ apresenta-

Trabalho realizado no Hospital da Beneficiência Portuguesa. Sâo Paulo, SP, Brasil.

Apresentado ao $17^{\circ}$ Congresso Nacional de Cirurgia Cardíaca, 6 e 7 de abril, 1990.

* Do Hospital da Beneficiência Portuguesa.

Endereço para separatas: Januário Souza. Rua Pará, 49. 01243 Sảo Paulo, SP, Brasil. 
SOUZA, J. M.; BERLINCK, M. F.; MOREIRA, M. G.; MARTINS, J. R. M.; MOREIRA, M. C. S.; OLIVEIRA, P. A. F.; SENRA, D. F.; OLIVEIRA, S. A. - Revascularização miocárdica em pacientes com idade igual ou superior a 70 anos. Rev. Bras. Cir. Cardiovasc., 5(3): 141-148, 1990.

vam, à epoca da operação, idade igual ou superior a 70 anos. Setenta e seis por cento desses pacientes (375) eram do sexo masculino. A idade variou de 70 a 87 anos, sendo $354(71,9 \%)$ entre 70 e 74 anos, $121(24,5 \%)$ entre 75 e 79 anos e $17(3,4 \%)$ entre 80 e 87 anos. A média das idades foi 73,3 anos (Tabela 1).

TABELA 1

MORTALIDADE POR FAIXAS ETÁRIAS

\begin{tabular}{lrrcccc}
\hline $\begin{array}{l}\text { Idade } \\
\text { (anos) }\end{array}$ & Nepac. & $\%$ & $\begin{array}{c}\text { Mortalidade } \\
\text { (Hosp./ 30 dias) }\end{array}$ & $\%$ & \\
\hline $70-74$ & 354 & 71,9 & 25 & 7,06 & \\
$75-79$ & 121 & 24,5 & 14 & 11,5 & p NS \\
$80-87$ & 17 & 3,4 & 3 & 17,6 & p NS \\
\hline TOTAL & 492 & & 42 & 8,5 & \\
\hline
\end{tabular}

Quanto à classe funcional para angina (de acordo com a Canadian Cardiovascular Society), $84 \%$ dos pacientes estavam em classes III e IV, $14,8 \%$ em classes I e II e apenas quatro $(0,8 \%)$ pacientes não apresentavam angina (Tabela 2).

TABELA 2

CLASSIFICAÇÃO FUNCIONAL DE ANGINA NO PRÉOPERATÓRIO DE ACORDO COM A CCS*

\begin{tabular}{lrr}
\hline $\begin{array}{l}\text { Classe funcional } \\
\text { (pré-operatório) }\end{array}$ & No pac. & \multicolumn{1}{c}{$\%$} \\
\hline Sem angina & 04 & 0,8 \\
I - II & 73 & 14,8 \\
III e IV & 415 & 84,3 \\
\hline
\end{tabular}

* Canadian Cardiovascular Society

O estudo cinecoronariográfico mostrou lesōes obstrutivas maiores que $70 \%$ em três artérias principais em $62,3 \%$ dos pacientes, em duas artérias em $31,4 \%$ e lesōes uniarteriais em $6,2 \%$. Desses pacientes, $76(15,4)$ apresentavam, também, lesão obstrutiva do tronco da coronária esquerda (Tabela 3). Vinte e oito pacientes foram operados durante a fase aguda do infarto do miocárdio, para revascularizaçāo isolada e/ou correção de defeitos mecânicos associados. Destes, quatro apresentavam rotura do septo interventricular e dois foram operados após reanimação cardiopulmonar, sendo levados para o centro cirúrgico sob massagem cardíaca.

Angina estável e instável foram as principais indicaçōes para a cirurgia de revascularizaçāo miocárdica. Operaçōes associadas foram realizadas em 54 pacientes, sendo em 30 para correção
TABELA 3

DISTRIBUIÇÄO DOS PACIENTES DE ACORDO COM O N DE ARTÉRIAS LESADAS

\begin{tabular}{ccr}
\hline$N^{0}$ artérias lesadas* & $N^{0}$ pac. & $\%$ \\
\hline 1 & 30 & 6,2 \\
2 & 154 & 31,4 \\
3 & 308 & 62,3 \\
\hline
\end{tabular}

* TCE $=76(15,4 \%)$ pacientes

TCE $=$ Tronco da Artéria Coronátia Esquerda

de aneurisma do ventrículo esquerdo, em 21 pacientes para correção de lesão valvar e em três pacientes foi realizada endarterectomia da carótida. Destes 492 pacientes, 465 eram operaçōes primárias; 27 eram reoperaçōes (Tabela 4 ).

TABELA 4

COMPARAÇÃO DA MORTALIDADE IMEDIATA DE ACORDO COM A CONDIÇÃO CLÍNICA PRÉ-OPERATÓRIA OU DA OPERAÇĀO REALIZADA

Condição Ne pac. operados $\begin{gathered}\text { Mortalidade } \\ \text { (Hosp./30 dias) }\end{gathered}$

\begin{tabular}{|c|c|c|c|c|}
\hline $\begin{array}{l}\text { Angina estável/ } \\
\text { instável }\end{array}$ & 410 & 21 & $5,12 \%$ & $p<0,0001$ \\
\hline I.A.M. & 28 & 13 & $46,04 \%$ & $p=0,002$ \\
\hline $\begin{array}{l}\text { Revasc. + op. } \\
\text { associada }\end{array}$ & 54 & 8 & $14,8 \%$ & \\
\hline Total & $492^{*}$ & 42 & $8,5 \%$ & \\
\hline
\end{tabular}

I.A.M. = infarto agudo do miocárdio

Revasc. = revascularização miocárdica

$\mathrm{OP} .=$ cperaçāo

* 27 Reoperações (5 óbitos - 18,5\%)

A quase totalidade dos pacientes foi operada com o auxílio de circulação extracorpórea e hipotermia sistêmica moderada $\left(28-32^{\circ} \mathrm{C}\right)$, com emprego do oxigenador de bolhas e, mais recentemente, oxigenador de membrana. Seis pacientes foram operados sem o emprego da circulaçăo extracorpórea. Soluçāo cardioplégica (solução St. Thomas 16 e soluçāo de Buckberg ') foi utilizada em aproximadamente $50 \%$ dos pacientes, sendo nos demais empregado o pinçamento aórtico intermitente.

Foram realizadas em média 2,7 pontes aortocoronárias por paciente. A artéria torácica interna (mamária) foi utilizada nesse grupo etário, com maior freqüência a partir de 1985 , passando de $5,3 \%$ dos pacientes operados até 1984 , para $48,6 \%$ dos pacientes operados no ano de 1989 (Tabela 7).

O controle pós-operatório tardio foi realizado diretamente no consultório, ou através de corres- 
SOUZA, J. M.; BERLINCK, M. F.; MOREIRA, M. G.; MARTINS, J. R. M.; MOREIRA, M. C. S.; OLIVEIRA, P. A. F.; SENRA, D. F.; OLIVEIRA, S. A. - Revascularizaçäo miocárdica em pacientes com idade igual ou superior a 70 anos. Rev. Bras. Cir. Cardiovasc., 5(3): 141-148, 1990.

pondência ou contacto telefonico com os pacientes, ou com o médico assistente.

Os dados foram analisados estatisticamente pelo método exato de Fischer e pelo Qui quadrado.

\section{RESULTADOS}

A mortalidade imediata (hospitalar ou 30 dias) foi de $8,5 \%$ (42/492). Entretanto, houve uma grande variação nos diferentes subgrupos de pacientes. Para os pacientes que foram operados para tratamento da angina estável ou instável, a mortalidade foi de $5,1 \%(21 / 410)$; para os pacientes operados na fase aguda do infarto do miocárdio foi de $46,4 \%$ $(13 / 28)$ e de $14,8 \%(8 / 54)$ naqueles pacientes com operaçōes associadas à revascularização miocárdica; essas diferenças foram estatisticamente significativas ( $p \leqslant 0,001$ e $p=0,002)$ (Tabela 4).

A mortalidade foi de $9,6 \%(36 / 375)$ para os pacientes do sexo masculino e de $5,1 \%(6 / 117)$ para os do sexo feminino, diferença não significativa. A mortalidade foi de $7,06 \%$ (25/354) para pacientes entre 70 e 74 anos, $11,5 \%$ (14/121) para aqueles entre 75 e 79 anos e de $17,6 \%(3 / 17)$ para os que tinham entre 80 e 87 anos; essas diferenças não foram estatisticamente significativas. Nos 27 pacientes que estavam sendo operados pela segunda vez, a mortalidade imediata foi de $18,5 \%(5 / 27)$ - $\mathrm{p}<0,0001$ (Tabela 4).

As causas de óbito mais freqüentes no pósoperatório imediato foram: síndrome de baixo débito cardíaco em dez pacientes, parada cardíaca em oito pacientes, insuficiência respiratória em seis, insuficiência renal aguda em cinco, acidente vascular cerebral em quatro, trombose mesentérica em dois e causas variadas nos outros sete pacientes (Tabela 8 ).

A mortalidade tardia foi de $6,5 \%$ (32/492), sendo que em 15 pacientes a causa foi cardíaca e em 17 não cardíaca.
Dezessete $(3,9 \%)$ pacientes não foram contactados recentemente. Dos 401 revisados, 374 estavam em classe funcional I ou II e 27 pacientes encontravam-se em classe funcional III ou IV (Tabela 5).

TABELA 5

RESULTADOS IMEDIATOS E TARDIOS DA REVASCULARIZAÇÄO MIOCÁRDICA ISOLADA, ASSOCIADA E NO IAM

\begin{tabular}{lrr}
\hline & Ne & $\%$ \\
\hline Óbitos imediatos (Hosp. - 30 dias) & 42 & 8,5 \\
Óbitos tardios & & \\
$\quad$ Causas cardíacas & 15 & 6,5 \\
$\quad$ Causas não cardíacas & 17 & \\
Vivos & & \\
G.F. I e II & 374 & 76,0 \\
G.F. III e IV & 27 & 5,5 \\
Sem controle recente & 17 & 3,9 \\
\hline
\end{tabular}

IAM - infarto agudo do miocárdio

\section{COMENTÁRIOS}

Várias publicaçōes recentes mostram a superioridade do tratamento cirúrgico sobre o tratamento clínico em muitos subgrupos de pacientes, seja quanto à sobrevida ou à melhoria dos sintomas, inclusive para os pacientes com idade igual ou superior a 65 anos ${ }^{10} \mathrm{com}$ lesão do tronco da coronária esquerda ou equivalente ${ }^{3,13,25}$, lesão proximal de três artérias, nos pacientes com baixa fração de ejeção e lesão multiarterial ${ }^{20} \mathrm{e}$ nos portadores de aneurisma do ventrículo esquerdo e de lesōes triarteriais '. É também observação freqüente a de que os pacientes idosos (mais de 70 anos), independentemente de qualquer outro fator, apresentam maior mortalidade pós-operatória imediata 2, 8, 11, 17, 19, 23 (Tabela 6).

TABELA 6

DADOS DA LITERATURA

\begin{tabular}{lccclr}
\hline Autor & Ano & Pacientes(Ne) & $\begin{array}{l}\text { Idade } \\
\text { (Anos) }\end{array}$ & Procedimento & Mort. hospitalar \\
\hline CABROL (2) & 1979 & 100 & $70-80$ & RM+CIR. VALVAR & $17,0 \%$ \\
ELAYDA (7) & 1984 & 1275 & $70-90$ & RM & $5,8 \%$ \\
DE VIVIE (5) & 1986 & 212 & $70-82$ & RM+CIR. VALVAR & $8,5 \%$ \\
SCHULTE (24) & 1987 & 201 & $70-82$ & RM+CIR. VALVAR & $8,5 \%$ \\
SOUZA (*) & 1989 & 492 & $70-87$ & RM+CIR. VALVAR+ & $8,5 \%$ \\
\hline
\end{tabular}

RM - Revascularização miocárdica; OP. fase AG. IM - operação na fase aguda do infarto

* Trabalho presente 
SOUZA, J. M.; BERLINCK, M. F.; MOREIRA, M. G.; MARTINS, J. R. M.; MOREIRA, M. C. S.; OLIVEIRA, P. A. F.; SENRA, D. F.; OLIVEIRA, S. A. - Revascularização miocárdica em pacientes com idade igual ou superior a 70 anos. Rev. Bras. Cir. Cardiovasc., 5(3): 141-148, 1990.

Em nossa casuística, observamos mortalidade imediata de $8,5 \%$ (42/492), quando analisado em conjunto; entretanto, houve grande variação de acordo com a condição clínica, a indicação cirúrgica, ou a operação realizada, sendo a mortalidade de $5,1 \%$ para os pacientes submetidos a revascularizaçăo miocárdica isolada, de $14,8 \%$ para aqueles que tiveram operaçōes associadas e de $46,4 \%$ para os operados durante a fase aguda do infarto do miocárdio, sendo essas diferenças altamente significativas (Tabela 4).

Os diferentes subgrupos etários $(70-74 a, 75-$ 79 a e $80-87$ anos) apresentaram diferentes taxas de mortalidade, mas essas diferenças não foram significativas (Tabela 1).

$\mathrm{RICH}$ et alii ${ }^{22} e$ TSAl et alii ${ }^{20}$ mostram baixa mortalidade imediata ( $4 \%$ e $5,2 \%$ ), em pacientes operados com mais de 80 anos, quando a revascularização miocárdica foi isolada, mas, quando foi associada à substituição valvar, a mortalidade subiu para $30 \%{ }^{20}$.

Os pacientes idosos (acima de 70 anos) apresentam uma incidência maior de doenças não cardíacas associadas, como lesōes do sistema nervoso central, aparelhos digestivo, respiratório e renal e insuficiência vascular periférica, o que aumenta os riscos de complicaçōes pós-operatórias, com aumento da mortalidade 6 , 13, 21, 24, 28.

Em dois pacientes de nossa série, foi impossível a passagem de balão intra-aórtico e, em outros dois, o balāo foi introduzido pela aorta ascendente, por apresentarem obstruçōes aorto-ilíacas. A presença de calcificação da aorta ascendente é fre- qüente, obrigando-nos, em alguns casos, a fazer a canulação para a circulação extracorpórea na croça ou numa das artérias femorais, sendo aconselhável, nesses casos, o uso de pontes seqüenciais ou em $Y$, diminuindo o número de anastomoses proximais na aorta, ou fazendo-as no tronco arterial braquiocefálico. $\mathrm{O}$ emprego da artéria torácica interna também é muito útil nessa situação. Até 1984, usávamos a artéria torácica interna em apenas $5,3 \%$ dos pacientes idosos, sendo mais utilizada a partir de entāo e, em 1989, essa porcentagem foi elevada para $48 \%$ dos pacientes operados (Tabela 7 ).

TABELA 7

FREQÜÊNCIA DA UTILIZAÇĀO DA ARTÉRIA TORÁCICA INTERNA (MAMÁRIA) NOS PROCEDIMENTOS CIRÚRGICOS DURANTE O PERIODDO ANALISADO

\begin{tabular}{lrcr}
\hline Período & No pac. & $\begin{array}{c}N^{0} \text { pac. } \\
\text { c/mamária }\end{array}$ & $P \%$ \\
\hline $1979-1984$ & 130 & 7 & 5,3 \\
1985 & 69 & 22 & 31,5 \\
1986 & 72 & 21 & 29,1 \\
1987 & 63 & 12 & 19,1 \\
1988 & 84 & 40 & 47,6 \\
1989 (out.) & 74 & 36 & 48,6 \\
\hline & 492 & 138 & 28,1 \\
\hline
\end{tabular}

A insuficiência renal aguda foi responsável por apenas um óbito no grupo de revascularizaçăo isolada e por quatro óbitos no grupo de pacientes operados na fase aguda do infarto do miocárdio. A

TABELA 8

ÓBITOS NOS DIVERSOS GRUPOS DE ACORDO COM OPERAÇŌES REALIZADAS

\begin{tabular}{|c|c|c|c|c|}
\hline Causas dos óbitos & $\begin{array}{c}\text { RM Isolada } \\
\quad(n=410) \\
N^{\circ} \text { pac. (\%) }\end{array}$ & $\begin{array}{c}\text { Op. assoc. à } R M \\
\left(\begin{array}{c}(n=54) \\
N^{9} \text { pac. (\%) }\end{array}\right.\end{array}$ & $\begin{array}{c}\text { RM fase aguda IM } \\
(n=28) \\
N^{2} \text { pac. (\%) }\end{array}$ & $\begin{array}{l}\text { Total (492) } \\
N^{\circ} \text { pac. (\%) }\end{array}$ \\
\hline SBDC & $4(0,9 \%)$ & $3(5,5 \%)$ & $3(10,7 \%)$ & $10(2 \%)$ \\
\hline Parada cardíaca & $5(1,2 \%)$ & $1(1,8 \%)$ & $2(7,1 \%)$ & $8(1,6 \%)$ \\
\hline Insuf. resp. & $4(0,9 \%)$ & $1(1,8 \%)$ & $1(3,5 \%)$ & $6(1,2 \%)$ \\
\hline IRA & $1(0,2 \%)$ & - & $14(14,2 \%)$ & $5(1 \%)$ \\
\hline AVC & $3(0,7 \%)$ & $1(1,8 \%)$ & - & $4(0,8 \%)$ \\
\hline T. mesentérica & $1(0,2 \%)$ & $1(1,8 \%)$ & - & $2(0,4 \%)$ \\
\hline Rotura da aorta & - & $1(1,8 \%)$ & - & $1(0,2 \%)$ \\
\hline IAM & - & - & $1(3,5 \%)$ & $1(0,2 \%)$ \\
\hline T. cardíaco & $1(0,2 \%)$ & - & - & $1(0,2 \%)$ \\
\hline Sepsis & $1(0,2 \%)$ & - & - & $1(0,2 \%)$ \\
\hline IMO & - & - & $2(7,1 \%)$ & $2(0,4 \%)$ \\
\hline Hipóxia POI & $1(0,2 \%)$ & - & - & $1(0,2 \%)$ \\
\hline
\end{tabular}

$\mathrm{SBCD}=$ síndrome baixo débito cardíaco; IRA = insuficiência renal aguda; $\mathrm{AVC}=$ acidente vascular cerebral; IMO = insuficiência múltiplos órgãos IAM = infarto agudo do miocárdio; RM = revascularizaçāo miocárdica; INS. RESP. = insuficiência respiratória; T. CARDÍACO = tamponamento cardíaco; T. MESENTÉRICA = trombose mesentérica; POI = pós-operatório imediato. 
SOUZA, J. M.; BERLINCK, M. F.; MOREIRA, M. G.; MARTINS, J. R. M.; MOREIRA, M. C. S.; OLIVEIRA, P. A. F.; SENRA, D. F.; OLIVEIRA, S. A. - Revascularizaçāo miocárdica em pacientes com idade igual ou superior a 70 anos. Rev. Bras. Cir. Cardiovasc., 5(3): 141-148, 1990.

síndrome do baixo débito cardíaco foi a causa mais freqüente de óbitos imediatos nessa série (10 casos), principalmente nos pacientes operados na fase aguda do infarto do miocárdio e nos que tiveram operaçōes associadas. A parada cardíaca foi também uma causa importante de mortalidade no pósoperatório imediato (oito casos), sendo considerada como causada por isquemia miocárdica quando nāo houve uma causa desencadeante. A insuficiência respiratória é uma complicaçāo pós-operatória grave, sendo mais freqüente nos pacientes tabagistas com doença pulmonar obstrutiva crônica. A preparaçăo pré-operatória com suspensāo do fumo e exercícios respiratórios pode diminuir a ocorrência dessa complicação.

$\mathrm{Na}$ avaliaçāo pré-operatória, especial atençāo deve ser dada ao exame neurológico do paciente. A presença de sopros caróticos indica realização de ecodopplercardiografia e, havendo lesão obstrutiva, deve ser feita corotidoangiografia. $O$ acidente vascular cerebral foi a causa de óbito em quatro pacientes.

Outra complicaçāo grave no pós-operatório é a isquemia mesentérica. A lavagem intestinal antes da operaçāo e a manutençāo de sonda gástrica e jejum até que se restabeleçam os movimentos peristálticos podem diminuir a incidência dessa complicaçăo (Tabela 8).

A influência do sexo sobre a mortalidade é variável. O sexo feminino apresenta mortalidade menor ${ }^{14}{ }^{18}$ ou maior ${ }^{4,17}$, segundo os autores. Em nossa série, a mortalidade foi maior nos pacientes do sexo masculino $9,6 \%(36 / 375)$ contra $5,1 \%$ (6/ 117) no feminino, mas essa diferença não teve significação estatística.

Os pacientes idosos operados apresentam, a longo prazo, um prognóstico pior em relaçāo aos pacientes jovens operados, mas o seu prognóstico a longo prazo (10-15 anos) é melhor em relação aos idosos nāo operados ${ }^{15,17}$.

O seguimento pós-operatório tardio estendeuse até dez anos, perdendo-se do controle apenas 17 pacientes. Ocorrerram 32 óbitos ao longo desse período, sendo que $46,8 \%$ foram por causas cardíacas. A probabilidade de sobrevida aos dez anos foi de $78,78 \pm 3,95 \%$ para os pacientes submetidos a revascularizaçāo miocárdica isolada, de $66,25 \pm 60 \%$ para aqueles que tiveram operaçōes associadas e de $39,4 \pm 14,86 \%$ (em oito anos) para os que foram operados durante a fase aguda do infarto do miocárdio pelos cálculos atuariais. Após a alta hospitalar, a evolução dos pacientes dos três grupos foi semeIhante, contrastando com as diferenças observadas quanto à mortalidade imediata (Gráfico 1).

\section{GRÁFICO 1 \\ REVASCULARIZAÇĀO MIOCÁRDICA NO PACIENTE IDOSO $(70 A)$}

JAN 1979 - OUT $1989-(N=492)$

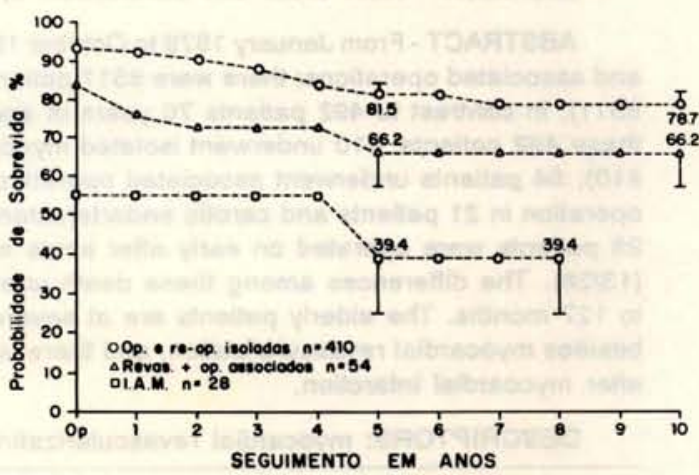

Concluindo, acreditamos que a idade avançada (acima de 70 anos) constitui um fator de risco para a cirurgia de revascularizaçāo miocárdica (Tabela 9). Entretanto, a adequada seleção pré-

TABELA 9

MORTALIDADE IMEDIATA NAS DIFERENTES CONDIÇŌES CLÍNICO-CIRÚRGICAS COMPARATIVAMENTE NOS PACIENTES COM IDADE MAIOR OU MENOR DE $7 O$ ANOS

\begin{tabular}{lllc}
\hline Condição & $\begin{array}{l}\text { Idade } \\
\text { (anos) }\end{array}$ & $n$ & $\begin{array}{c}\text { Mortalidade } \\
\text { (Hosp./30 dias) }\end{array}$
\end{tabular}

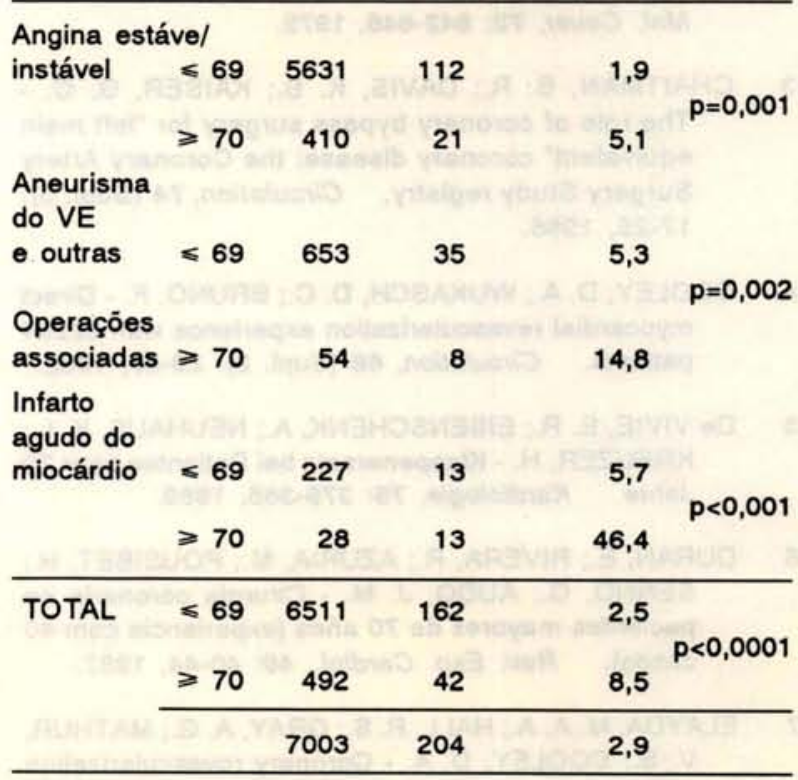

operatória, além de cuidados intra e pós-operatórios, permitiram a esses pacientes ter melhor qualidade de vida e, provavelmente, um aumento da expectativa de vida. 
SOUZA, J. M.; BERLINCK, M. F.; MOREIRA, M. G.; MARTINS, J. R. M.; MOREIRA, M. C. S.; OLIVEIRA, P. A. F.; SENRA, D. F.; OLIVEIRA, S. A. - Revascularização miocárdica em pacientes com idade igual ou superior a 70 anos. Rev. Bras. Cir. Cardiovasc., 5(3): 141-148, 1990.

RBCCV 44205-117

SOUZA, J. M.; BERLINCK, M. F.; MOREIRA, M. G.; MARTINS, J. R. M.; MOREIRA, M. C. S.; OLIVEIRA, P. A. F.; SENRA, D. F.; OLIVEIRA, S. A. - Myocardial revascularization in patients 70 years of age and older. Rev. Bras. Cir. Cardiovasc. 5(3): 141-148, 1990.

ABSTRACT - From January 1979 to October 1989, 7003 patients underwent myocardial revascularization and associated operations; there were 6511 patients under the age of 70 , with early mortality of $2.5 \%$ (162) $6511)$, in contrast to 492 patients 70 years of age or older with early mortality of $8,5 \%(42 / 492)$. Among these 492 patients, 410 underwent isolated myocardial revascularization with early mortality of $5.1 \%(21)$ 410); 54 patients underwent associated operatins (left ventricle aneurysmectomy in 30 patients, valvular operation in 21 patients and carotid endarterectomy in 3 patients) with early mortality of $14.8 \%(8 / 54)$ and 28 patients were operated on early after acute myocardial infarction and the early mortality was $46.4 \%$ (13/28). The differences among these death-rates were significant. A $96.1 \%$ follow-up was obtained up to 127 months. The elderly patients are at severe risk mainly when they undergo associated operations besides myocardial revascularization, and there is a more significant risk when they are operated on early after myocardial infarction.

DESCRIPTORS: myocardial revascularization, surgery, elderly patients.

\section{REFERÊNCIAS BIBLIOGRÁFICAS}

1 BUCKBERG, G. D. - Strategies and logic of cardioplegic delivery to prevent, avoid, and reverse ischemic and reperfusion damage. J. Thorac. Cardiovasc. Surg., 93: $127-139,1987$.

2 CABROL, C. J.; BRUSAID, J. J.; DOUMEIX, G.; BLANC, J. A.; MORIN, B. - Risques et résultats à moyen terme du replacement valvulaire aortique pour stenose calcifiée chez 100 sujets de plus de 70 ans. Arch. Mal. Couer, 72: 842-848, 1979.

3 ChAITMAN, B. R.; DAVIS, K. B.; KAISER, G. C. The role of coronary bypass surgery for "left main equivalent" coronary disease: the Coronary Artery Surgery Study registry, Circulation, 74 (Supl. 3): 17-25, 1986.

4 COOLEY, D. A.; WUKASCH, D. C.; BRUNO, F. - Direct myocardial revascularization experience with 22284 patients. Circulation, 68 (Supl. 2): 20-29, 1982.

5 De VIVIE, E. R.; EISENSCHENK, A.; NEUHAUS, K. L.; KREUZER, H. - Klappenersatz bei Patienten über 70 Jahre. Kardiologie, 75: 379-385, 1986.

6 DURAN, E.; RIVERA, R.; AZURIA, M.; POUSIBET, H.; SENNO, G.; AUDO, J. M. - Cirurgia coronaria en pacientes mayores de 70 años (experiencia com 40 casos). Rev. Esp. Cardiol., 40: 40-44, 1987.

7 ELAYDA, M. A. A.; HALL, R. S.: GRAY, A. G.; MATHUR, V. S.; COOLEY, D. A. - Coronary revascularization in the elderly patient. J. Am. College Cardiol., 3: 1398-1402, 1984.

FARO, R. S.; GOLDEN, M. D.; JAVID, H.; SERRY, G. - Coronary revascularization in septuagenarians. $J$. Thorac. Cardiovasc. Surg., 86: 616-620, 1985.
9 FAXON, D. P.; MCCABE, C. H,; RYAB, T. J.; MYERS W. O.; DAVIS, K. B.; SCHAFF, H. V. - The value of left ventricular aneurysm resection in the management of patients with aneurysm undergoing bypass surgery (CASS). Circulation, 68 (Supl. 3): 115 $124,1983$.

10 GERSH, B. J. KRONWAL, R. A.; SCHAFF, H. V. Comparison of coronary artery bypass surgery and medical therapy in patients 65 years of age or older: a monrandomized study from the coronary artery study (CASS) registry. N. Engl. J. Med., 313: 217 . 224, 1985.

11 HORNEFFER, P.J.; GARDNER, T. J.; MANOLIO, T. A.; HOFF, S. J. - The effects of age on outcome after coronary bypass sugery. Circulation, 76 (Supl. 5) 6-12, 1987.

12 IBGE - Recenseamento Geral do Brasil. Vol. 1, Tomo 2. 1980.

13 JEFFERY, D. L.; VIJAYANAGAR, R. R.; BOGNOLO, D. A.; ELKSTEIN, P. F. - Coronary bypass for left main disease in patients over 70 years of age. J. Cardiovasc. Surg., 26: 212-216, 1985.

14 JEFFERY, D. L.; VIJAYANAGAR, R. R.: BOGNOLO, D. A.; ELKSTEIN, P. F. - Results of coronary bypass surgery in elderly women. Ann. Thorac. Surg., 42: 550-553, 1986.

15 JOHNSON, W. D.; BRENOWITZ, J. B.; KAYSER, K. L. - Factors influencing long-term (10 year to 15 year) survival after a successful coronary artery bypass operation. Ann. Thorac. Surg. 48: 19-25, 1989.

16 JYNGE, P.; HEARSE, D. J.; FEUVRAY, D. - The St. Thomas' Hospital cardioplegic solution: a characterization in two species. Scand. J. Thorac. Cardiovasc. Surg. 22 (Supl. 30): 1-28, 1981. 
SOUZA, J. M.; BERLINCK, M. F.; MOREIRA, M. G.; MARTINS, J. R. M.; MOREIRA, M. C. S.; OLIVEIRA, P. A. F.; SENRA, D. F.; OLIVEIRA, S. A. - Revascularizaçảo miocárdica em pacientes com idade igual ou superior a 70 anos. Rev. Bras. Cir. Cardiovasc., 5(3): 141-148, 1990.

17 LOOP, F. D.; LYTLE, B. W.; COSGROVE, D. M.; GOORMASTIC, M.; TAYLOR, P. C. - Coronary artery bypass graft surgery in the elderly. Clev. Clin. J. Med., 55: 23-24, 1988.

18 MONTAGNE, N. T.; KOUCHOUKOS, N. T.; WILSON, T. A. S.; BENNETT, A. L. - Morbidity and mortality of coronary bypass graffing in patients 70 years of age and older. Ann. Thorac. Surg. 39: 552-557, 1985.

MORAES, C. R.; RODRIGUES, J. V.: TENÓRIO, E.; GOMES, C. - Influência da idade na morbidade e mortalidade da cirurgia arterial coronária. Arq. Bras. Cardiol., 43: 161-163, 1989.

20 PASSAMANOI, E.; DAVIS, K. B.; GILleSPIE, M. J. - A randomized trial of coronary artery bypass surgery: survival of patients with a low ejection fraction. $N$. Engl. J. Med., 312: 1665-1671, 1985.

21 RICH, M. W.; KELLER, A. J.; SCHECHTMAN, K. B. MARSHALL, W. G.; KONCHOUKOS, N. T. - Morbility and mortality of coronary bypass surgery in patients 75 years of age or older. Ann. Thorac Surg., 46: 638-644, 1988.

RICH, M. W.; SANDZA, M. D.; KLEIGER, R. E.; CONNORS, J. P. - Cardiac operation in patients over 80 years of age. J. Thorac. Cardiovasc. Surg., 90: 56-60, 1985.

23 ROBERTS, A. S.; WOODHALL, D. D.; CONTI, C. R. Mortality-morbility and cost-accouting related to coronary artery bypass graft in the elderly. Ann. Thorac. Surg., 39: 426-432, 1985.

24 SCHULTE, H. D.; BIRCKS, W.; KRIAN, A.; MATEJIC B. - Coronary and valvular surgery in elderly patients ( $\geqslant 70$ years). Thorac. Cardiovasc Surgeon, 35: 143-147, 1987.

TALANO, J. V.; SCANLON, P. A.; MEADOWS, W. R.; KAHNN PIFFARE, R.; GUNNAR, R. M. - Influence of surgery on survival in 145 patients with left main coronary artery disease. Circulation, 51/52 (Supl. 1): $1105-1111,1975$

26 TSAI, T. P.; MALLOF, J. M.; GRAY, R.S.; CHAUX, A. KASS, R. M. - Cardiac surgery in the octogenarian. J. Thorac. Cardiovasc. Surg., 91: 924-928, 1986.

\section{DISCUSSĀO}

\section{DR. JOSÉ CARLOS IGLÉZIAS \\ São Paulo, SP}

Em período pouco superior a uma década, a operação de revascularização do miocárdio no paciente idoso evoluiu muito, não só devido aos avanços técnicos, como ao aprimoramento das equipes cirúrgicas e à melhoria na proteção miocárdica. Previamente, os pacientes com idade igual ou superior a 65 anos tinham suas opera- çōes contra-indicadas, levando-se em consideração, quase que tão somente, o fator idade. $O$ trabalho apresentado pelo $\mathrm{Dr}$. Januário e colaboradores traz uma importante contribuição ao estudo da revascularizaçāo do idoso em nosso meio, não só pela casuística (492 pacientes idosos/7003, número global de pacientes da série), como pelos resultados do seguimento tardio (10 anos) mostrando que se trata de recurso válido e que, pela análise criteriosa dos fatores de risco previamente, deve ser extendida aos portadores de coronariopatia, independentemente da idade. Pela leitura do trabalho, podemos destacar, comparativamente a outras casuísticas, que a mortalidade operatória é diferente na dependência do subgrupo estudado. Embora nāo haja significância estatística quanto aos subgrupos etários, a mortalidade hospitalar é diferente e crescente, quando comparamos o subgrupo dos pacientes que se submeteram a revascularização isolada com os do subgrupo que tiveram outro procedimento associado, tal como a aneurismectomia do ventrículo esquerdo, e que, por sua vez, apresentam mortalidade bem menor $(14,8 \%)$ do que aqueles do subgrupo onde foram incluídos os pacientes operados na fase aguda do infarto do miocárdio $(46,4 \%)$. Como contribuição, sugerimos que, nos próximos pacientes operados, sejam monitorizados alguns parâmetros com a finalidade de, através de uma análise discriminatória, estabelecer aqueles que exibem algum grau de significância estatística e possam permitir um prognóstico para a mortalidade operatória. $O$ estudo seria ainda mais proveitoso se, por intermédio de um modelo matemático, pudesse ser realizada uma análise multivariável que permitisse identificar as associaçōes pré-operatórias de maior risco para morbidade e mortalidade hospitalar. Baseado nestes fatos, tentar-se-ia, diante de situaçōes específicas para cada paciente, conduzir o ato operatório no sentido de mudar a evolução natural da moléstia e diminuir a incidência de intercorrências pósoperatórias, reduzindo, com isto, a permanência hospitalar e, portanto, o custo do procedimento. Pela análise da curva atuarial, percebemos que, passado o período de risco inicial, os respectivos subgrupos mantêm uma expectativa de vida praticamente constante. Como ficou provado na análise do trabalho, os pacientes idosos apresentam mortalidade operatória maior que aqueles com idade inferior a 65 anos, assim como são acometidos por maior número de intercorrências: neurológicas, pulmonares, renais, etc.; no entanto, o objetivo e os benefícios da revascularização do miocárdio também a eles devem ser extendidos, quais sejam: evitar a morte súbita, o infarto do miocárdio, aliviar a angina e, dentro do possível, prolongar a vida. 
SOUZA, J. M.; BERLINCK, M. F.; MOREIRA, M. G.; MARTINS, J. R. M.; MOREIRA, M. C. S.; OLIVEIRA, P. A. F.; SENRA, D. F.; OLIVEIRA, S. A. - Revascularização miocárdica em pacientes com idade igual ou superior a 70 anos. Rev. Bras. Cir. Cardiovasc., 5(3): 141-148, 1990.

DR. SOUZA

(Encerrando)

Agradeço os comentários e as sugestōes do Dr. Iglézias. Acredito que o nosso trabalho demonstrou, claramente, que os pacientes com idade superior a 70 anos apresentam um risco cirúrgico imediato (hospitalar ou 30 dias) maior que os mais jovens. Esse risco foi mais acentuado quando foram realizadas operaçōes associadas (aneurismectomia do
VE, cirurgia valvar), ou quando a operação foi durante a fase aguda do infarto miocárdico. Os pacientes operados de maneira eletiva apresentaram mortalidade baixa. Outro dado importante a ressaltar é a boa evoluçăo a longo prazo (semeIhante nos vários subgrupos), confirmando dados da literatura, que mostram evoluçāo tardia melhor nos pacientes submetidos a revascularização miocárdica nessa faixa etária (maior que 70 anos) que nos não operados. 